\title{
Protective effects of black bamboo leaves on the glucose- induced toxicity in Caenorhabditis elegans
}

\author{
Eun Hye Park ${ }^{1}$, Hong Jun Kim ${ }^{2}$, Dong Seok Cha ${ }^{1 * \mathbb{}}$ \\ ${ }^{1}$ College of Pharmacy, Woosuk University, Jeonbuk 55338, Republic of Korea \\ ${ }^{2}$ Department of Korean Medicine, Woosuk University, Jeonbuk 55338, Republic of Korea
}

\section{A R T I C L E I N F O}

Article Type:

Original Article

\section{Article History:}

Received: 15 June 2020

Accepted: 20 August 2020

\section{Keywords:}

Phyllostachys nigra

Black bamboo

Glucose toxicity

Diabetes mellitus

Caenorhabditis elegans

\begin{abstract}
A B S T R A C T
Introduction: Previous studies have suggested that high glucose (HG) condition shortens the lifespan of worms by increasing glycolytic flux followed by accelerating reactive oxygen species (ROS) production, termed glucose toxicity. This study was designed to investigate the protective effects of the leaves of black bamboo (Phyllostachys nigra var. henosis) on the HGinduced toxicity using Caenorhabditis elegans model system.

Methods: To determine the effect of black bamboo leaf extract (BLE) against HG-induced toxicity, lifespan assay was carried out with wild-type and daf-16 null mutant worms under $2 \%$ glucose condition. The involvement of DAF-16 was further confirmed by observing fluorescence signal of transgenic mutant carrying DAF-16::GFP transgene. ROS levels and glucose concentration of worms were analyzed using fluorescent probe $\mathrm{H}_{2}$ DCF-DA and glucose meter, respectively. Nile-red staining was carried out to evaluate the lipid storage of worms. Intercellular lipid accumulation was measured using Oil-Red $\mathrm{O}$ staining method.

Results: BLE strongly extended the lifespan of worms under not only normal culture condition but also HG condition. Our additional studies suggested that DAF-16 activation was responsible for BLE-mediated longevity and protective action against glucose toxicity. In addition, HG-fed worms showed increased ROS generation, and it was completely normalized by BLE treatment. Moreover, BLE reduced body glucose concentration and lipid accumulation in HG-fed worms. We further confirmed the inhibitory effect of BLE on the lipid storage under HG condition using 3T3-L1 adipocytes.

Conclusion: These therapeutic values of BLE on the glucose toxicity raise the possibility that BLE might have beneficial effects on the pathogenesis of diabetes mellitus.
\end{abstract}

Implication for health policy/practice/research/medical education:

Black bamboo leaves showed potent protective potential against glucose toxicity, and thereby could be suggested as a candidate in the development of new therapeutic agent for diabetic mellitus and its complications.

Please cite this paper as: Park EH, Kim HJ, Cha DS. Protective effects of black bamboo leaves on the glucose-induced toxicity in Caenorhabditis elegans. J Herbmed Pharmacol. 2021;10(2):226-231. doi: 10.34172/jhp.2021.25.

\section{Introduction}

Type 2 diabetes mellitus (T2DM) is a kind of metabolic disease caused by maintained long-term hyperglycemia resulting from insulin resistance (1). Previous studies have suggested that a hyperglycemic condition brings about an elevated production of reactive oxygen species (ROS) (2). Under high glucose conditions, glycation of protein and activation of mitochondrial enzymes are highly accelerated, resulting in the promotion of excessive ROS formation. In addition to increased ROS generation, hyperglycemia is also known to attenuate NADPH and glutathione levels, which contribute to enhanced sensitivity to oxidative stress (3). Therefore, a strategy focused on diminishing oxidative stress may help prevent or delay the onset of T2DM.

Insulin resistance, a core feature of T2DM, is closely associated with obesity, and indeed, about $90 \%$ of T2DM is attributed to excess weight (4). In the case of obese patients, the lipid storage capacity of adipocyte can be exceeded, resulting in abnormal fat accumulation in nonadipose tissues such as muscle, liver, and pancreatic islets (5). Excess accumulation of lipids in these tissues is thought 
to cause insulin resistance through cell-autonomous mechanisms and induction of inflammatory responses (6). Both of these excessive oxidative stress and abnormal lipid accumulation are closely involved in the diabetic complications by increasing the risk of macrovascular and microvascular disorders.

The leaves of Phyllostachys nigra var. henosis, which is also known as black bamboo, a kind of bamboo species, has been used for fever as a traditional remedy in China and Korea. Phytochemical studies have indicated that the leaves of this plant contain high amounts of polyphenolic compounds with potent antioxidant capacity (7). Many pharmacological studies on this plant have revealed that it has hepatoprotective (8), anti-melanogenic (9), and anti-inflammatory (10) properties. Also, isolated flavone compounds from this plant such as luteolin 6-C-(6"-Otrans-caffeoylglucoside), inhibit both aldose reductase activity and advanced glycation end products (AGEs) formation (11). Recently, a protective effect on diabetic nephropathy has been reported on other bamboo species (12). Based on these backgrounds, herein we tried to dissect the therapeutic potential of black bamboo leaves on the glucose toxicity using Caenorhabditis elegans model system.

\section{Materials and Methods}

Plant material and extraction

The plant materials were purchased from Kwangmyeongdang Medicinal Herbs (Ulsan, South Korea) in March 2015. The plant was identified by Professor Dae Keun Kim (Laboratory of Pharmacognosy, Woosuk University) and the voucher specimen was deposited in the herbarium of Department of Korean Medicine, Woosuk University. The dried leaves of the plant $(500 \mathrm{~g})$ were extracted with 1000 $\mathrm{mL}$ of hot water for 3 hours. The resultant extract (BLE) was concentrated with a rotary evaporator and the residual liquid was lyophilized to a powder form.

Cell culture and Oil-red $\mathrm{O}$ staining

3T3-L1 preadipocytes were obtained from America Type Culture Collection (ATCC; Rockville, MD, USA) and maintained in DMEM (Dulbecco's Modified Eagle Medium, Thermo Scientific) containing 10\% NBCS (3T3$\mathrm{L} 1$ cells) at $37^{\circ} \mathrm{C}$ in $5 \% \mathrm{CO}_{2}, 95 \%$ air. Intercellular lipid accumulation was measured using the Oil-Red $\mathrm{O}$ staining method. Differentiated 3T3-L1 preadipocytes were washed twice with cold distilled water and fixed in $10 \%$ formalin at room temperature. After 1 hour, the cells were washed with $60 \%$ isopropanol and stained with $3 \mathrm{mg} / \mathrm{mL}$ Oil-Red $\mathrm{O}$ (in 60\% isopropanol) another 1 hour. Then the cells were rewashed with distilled water. 3T3-L1 preadipocytes were eluted with $100 \%$ isopropanol and observed under a microscope (Olympus, Japan). The absorbance was measured at $510 \mathrm{~nm}$ using a spectrophotometer.

Caenorhabditis elegans maintenance, lifespan assay, and fluorescence microscopy

Caenorhabditis elegans strains including Bristol N2 (wild type), GR1307 (mgDf50), TJ356 (zIs356) and Escherichia coli OP50 were obtained from the Caenorhabditis Genetic Center (CGC; University of Minnesota, Minneapolis, $\mathrm{MN})$. The worms were grown on nematode growth medium (NGM) agar plate with E. coli OP50 (OD600 0.7 ), and the sample plate was prepared by adding BLE stock solution into autoclaved NGM plates at $50^{\circ} \mathrm{C}$. For lifespan assay, age-synchronized worms were transferred to normal or $2 \%$ glucose-containing NGM plate in the absence or presence of $6.25,12.5$ and, $25 \mu \mathrm{g} / \mathrm{mL}$ of BLE. Test worms were considered dead when they failed to respond to prodding with the tip of platinum wire (13). The worms were transferred to fresh NGM plate every two days. For fluorescence microscopy observation, TJ356 mutant worms carrying DAF-16::GFP transgene were anesthetized with $2 \%$ sodium azide. Then the GFP fluorescence was directly observed under an uplight fluorescence microscope (Eclipse Ci, Nikon, Japan).

\section{Measurement of glucose concentration}

Glucose concentration was determined after eight days of incubation on the NGM plate containing $2 \%$ glucose at $20^{\circ} \mathrm{C}$. Harvested worms were washed three times using M9 and then homogenized on ice with a polytron homogenizer (Bio-Gen PRO200 Homogenizer, PRO Scientific, USA). Total protein content of homogenates was quantified using the Bradford assay. Glucose concentration inside of worms was measured using a glucose meter (Accu-Chek active kit, Roche Diagnostics GmbH, Germany).

\section{Analysis of intracellular ROS and lipid storage}

Intracellular ROS in the nematodes was measured using 2,7-dichorodihydrofluoroscein diacetate ( $\left.\mathrm{H}_{2} \mathrm{DCF}-\mathrm{DA}\right)$ as described previously (14). Lipid storage in C. elegans was quantified with Nile red staining. Nile red was fed to worms by mixing it with $E$. coli in the ratio 1:250. The worms of adult day one were transferred onto Nile red containing NGM plate and grown for four days at $25^{\circ} \mathrm{C}$. Prior to microscopy observation, worms were washed two times using M9 and then anesthetized with $2 \%$ sodium azide and mounted onto $2 \%$ agarose pads. The content of lipid staining was estimated using a fluorescence microscope (SMZ1500, Japan). Photographs of stained worms were further analyzed using Image J software.

\section{Data analysis}

The data from the lifespan assay were plotted using Kaplan-Meier analysis, and statistical significance was analyzed by the log-rank test. Other data were presented as mean \pm standard deviation. Statistical significance of differences between the control and treated groups were analyzed by one-way analysis of variance (ANOVA) followed by the Scheffe's test. Differences were considered 
significant if the $P$ value was less than 0.1 .

\section{Results}

BLE extends lifespan of nematode under high glucose conditions via accelerating DAF-16 activation

To determine the possible protective action of BLE against glucose-induced toxicity, lifespan assay was carried out with wild-type worms, incubated on NGM agar plate containing $2 \%$ glucose. We found that the lifespan of HGfed worms was significantly diminished, compared to that of worms under normal conditions. The BLE treatment effectively recovered this glucose toxicity-induced shortened lifespan (Figure 1B). Then, to determine whether BLE might also affect longevity, we performed lifespan assay again under normal conditions. As can be seen in Figure 1A, BLE-treatment significantly prolonged the lifespan of worms in a dose-dependent manner. Next, to understand underlying genetic mechanism of BLE-mediated protection, we tested the effect of BLE on the glucose toxicity again using daf-16 null mutants. As shown in Figure 1C, BLE failed to extend the lifespan of mutant worms under HG condition, suggesting DAF-16 is required for the BLE-mediated protection against glucose toxicity. We further investigated the involvement of DAF16 using mutant worms carrying daf-16::gfp transgene. Analysis of fluorescence signals from transgenic mutant let us know that DAF-16 activation is diminished under HG condition, compared with normal conditions (Figure 1D). Importantly, BLE treatment could accelerate the nuclear translocation of DAF-16, confirming that DAF-16 activation contributes to BLE's protective action.

BLE reduces glucose and ROS levels under high glucose condition

Excessive glucose supply increases glucose concentration inside of worms, and this elevated glucose level is believed to cause lifespan shortening (15). Here in this study, we examined whether BLE could regulate glucose concentration of worms using worm homogenates. As similar to previous report, glucose concentration was elevated significantly in HG-fed worms. Also, BLE treatment reduced the glucose concentration of worms from $26.3 \mathrm{mg} / \mathrm{dL}$ to $10.3 \mathrm{mg} / \mathrm{dL}$ (Figure 2A). Then, we tested whether BLE might alter intracellular ROS levels under HG condition using $\mathrm{H}_{2}$ DCF-DA probe. Because, $\mathrm{HG}$ condition is known to contribute to generate excessive ROS, followed by inducing oxidative stress. We showed that BLE treatment efficiently down-regulated the HGinduced increased ROS levels (Figure 2B).

BLE attenuates high glucose-induced lipid storage

Since glucose surplus could be transformed into lipid, we further investigated the effects of BLE on the lipid storage under $\mathrm{HG}$ condition both in in vitro and in vivo. To assess the effect of BLE on the lipid storage in adipocytes, we stained the cells using Oil-Red O. In this study, considerable lipid accumulation was observed in the HG-treated 3T3-L1 adipocytes. While the stained area
A

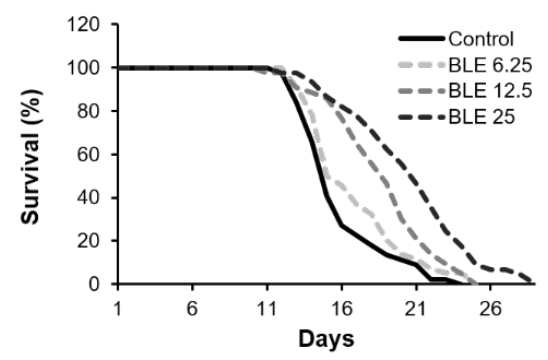

C

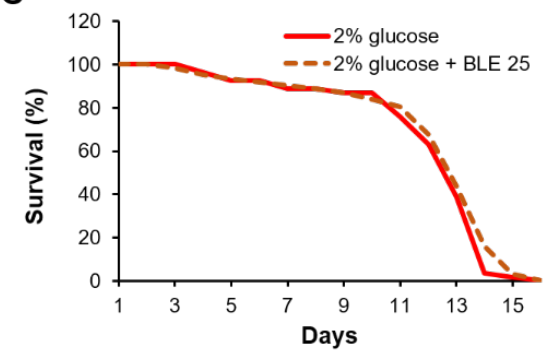

B



D

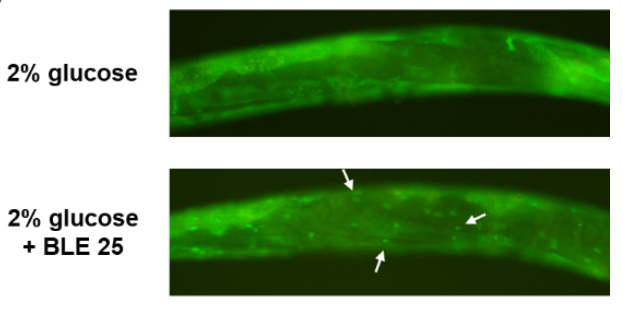

Figure 1. Effects of black bamboo leaf extract (BLE) on the lifespan of worms under normal and high glucose (HG) condition. Using wild-type N2 worms, lifespan assay was performed under normal culture condition (A) and HG condition (B) in the presence of absence of BLE (6.25, 12.5, $25 \mu \mathrm{g} / \mathrm{ml})$. (C) Lifespan assay was further performed with GR1307 strain (daf-16 null mutant) under HG condition. (D) The translocation of DAF-16 was visualized under fluorescence microscope using TJ356 strain which carries DAF-16::GFP transgene. 
A

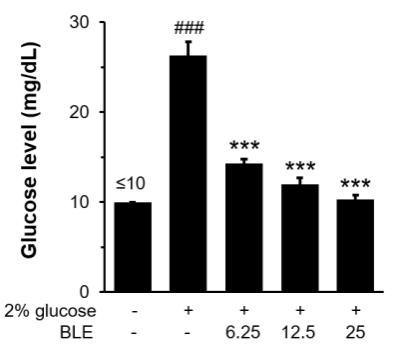

B

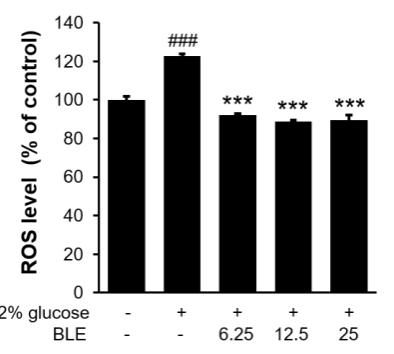

Figure 2. Effects of black bamboo leaf extract (BLE) on the glucose concentrations and intracellular reactive oxygen species (ROS) levels of high glucose (HG)-fed worms. To measure glucose concentration of worms, homogenized lysate was prepared with about 100,000 worms for each sample. (A) Body glucose concentration of HGfed worms was analyzed using glucose meter. (B) Intracellular ROS level was quantified spectrophotometrically using fluorescent probe, $\mathrm{H}_{2} \mathrm{DCF}$ DA at excitation $490 \mathrm{~nm}$ and emission $510-570 \mathrm{~nm}$. The plate was read at 60 min after treatment of $\mathrm{H}_{2}$ DCF-DA. Data are expressed as the mean $\pm \mathrm{SD}$. of three independent experiments. Differences between groups were statistically analyzed by one-way ANOVA. $P<0.001$ compared to control, while ${ }^{* * *} P<0.001$ compared to $2 \%$ glucose-treated groups.

was strongly reduced by BLE supplementation with no influence on cell morphology or viability. BLE-mediated decreased accumulation of lipid droplets was confirmed by measuring the absorbance of Oil-Red $\mathrm{O}$ (Figure $3 \mathrm{~A}$ and $3 \mathrm{~B})$. Then, we tested the anti-lipogenic effect of BLE again by Nile red staining using $C$. elegans. Consistent with the result from adipocytes, BLE attenuated the HG-induced lipid accumulation of worms, dose dependently (Figure $3 \mathrm{C}$ and $3 \mathrm{D})$.

\section{Discussion}

The conserved gene expression features between human diabetes and HG-fed worms have been confirmed by previous studies (16). Worms have conserved insulin signaling system called insulin/IGF-1 signaling pathway (IIS pathway). Both aging process and hyperglycemic condition are closely interconnected via this conserved transcriptional link (17). Blocking the IIS pathway prolongs the lifespan, while up-regulation of this signaling in response to excess glucose diet leads to lifespan shortening. In C. elegans, therefore, glucose toxicity can be represented as a simple phenotype exhibiting a reduced lifespan (18). As in humans, a high glucose diet is also known to cause decreased functional ability of worms (19).

The IIS pathway negatively regulates the FOXO transcriptional factor, a core regulator of stress responses, metabolism, and apoptosis (20). Similar to mammals, DAF16, a FOXO homolog in C. elegans, promotes longevity and stress tolerance. Previously, Lee's group reported that diminished DAF-16 activities were responsible for HG-induced shortened lifespan in C. elegans (15). In this study, BLE was able to completely rescue the decrease in lifespan caused by HG exposure via activating DAF-16. In addition, under HG condition, BLE treatment efficiently ameliorated the glucose concentration of worms. Together, these results imply that BLE's protective action against glucose toxicity might be attributed to decreased IIS pathway signals resulted in activation of DAF-16. Then, how does BLE regulates glucose levels? It is not clear but it could be suggested that BLE might regulate body glucose levels by affecting glucose uptake or glucose utilization.

\section{A}
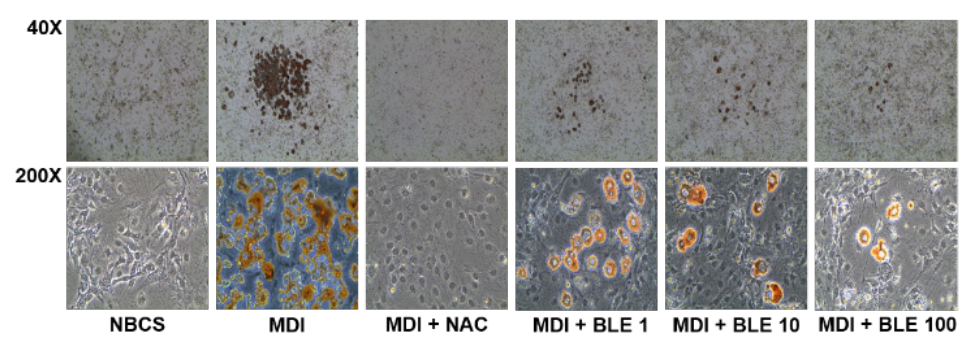

B



C
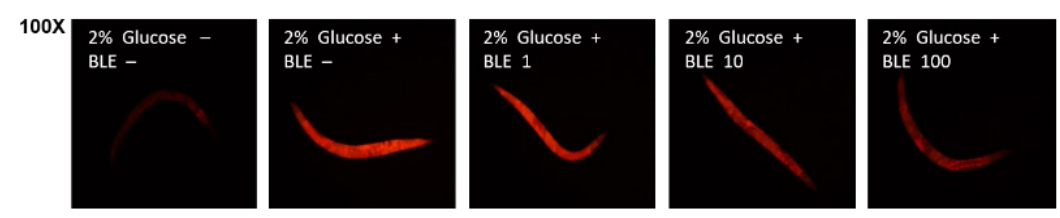

D

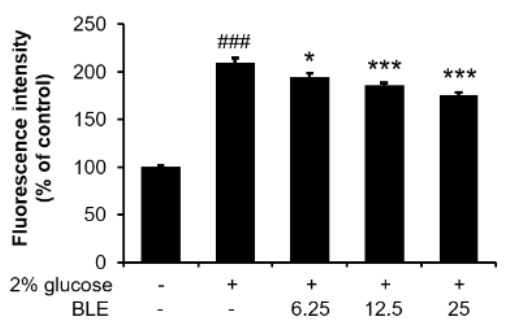

Figure 3. Effects of black bamboo leaf extract (BLE) on the lipid accumulation in 3T3-L1 adipocytes and worms under high glucose (HG) condition. (A) Microscopic images of Oil-Red O stained 3T3-L1 adipocytes. A standard differentiation mixture (MDI) induced adipogenic differentiation of preadipocytes. NBCS (non-treatment), NAC (10 mM) and BLE-treated group (1, 10,100 $\mathrm{gg} / \mathrm{mL})$ for 8 days during differentiation. (B) Lipid accumulation was further quantified spectrophotometrically at $510 \mathrm{~nm}$. (C) Fluorescent images of lipid of worms after Nile red staining. (D) Fluorescence intensity of worms was quantified using Image $\mathrm{J}$ software by determining average pixel intensity. Data are expressed as the mean \pm S.D. of three independent experiments. Differences between groups were statistically analyzed by one-way ANOVA. ${ }^{\# \#} P<0.001$ compared to control, while ${ }^{*} P<0.1$ and ${ }^{* * *} P<0.001$ compared to $2 \%$ glucose-treated groups. 
In addition to DAF-16, a reduced IIS pathway is also known to activate other transcriptional factors such as HSF-1 and SKN-1, a mammalian Nrf2 homolog. Recent evidence from the liver injury model demonstrated that polyphenol mixtures of black bamboo induce Nrf2 activation under oxidative stress conditions (8). Several studies have shown that HG condition shortens the lifespan of worms by increasing glycolysis, subsequently causing mitochondrial ROS generation through aerobic catabolism (21). Thus, BLE's strong antioxidant capacity and reinforcing antioxidant defense mechanism by Nrf2 activation might be expected to contribute to BLEmediated protection against HG-induced oxidative stress. Indeed, we showed that HG-induced elevated ROS levels were remarkably blocked by BLE, which is hard to explain solely by direct scavenging activity.

High glycolytic flux resulting in decreased glyoxalase-1 activity is known to lead to abnormal methylglyoxal production (21). The methylglyoxal, a highly reactive metabolite of glucose, causes non-enzymatic glycation of proteins or lipids to form AGEs, which is known as one of the critical factors in the pathogenesis of diabetes and aging (22). Previous in vitro study has revealed that flavone compounds from black bamboo can act as an AGEs inhibitor. Together, these results provide evidence that BLE-mediated protection against glucose toxicity might be attributed to reduced oxidative stress via downregulation of the IIS pathway.

The excessive fat deposition and the subsequent lipotoxicity plays a crucial role in promoting insulin resistance, and thus, attributes $\beta$-cell dysfunction in the pathogenesis of type 2 diabetes (23). Our Oil-Red O staining data showed that accumulation of lipid into the adipocytes was markedly suppressed by BLE treatment under HG conditions. Early studies indicated that glucose feeding also increases fat accumulation in C. elegans (24). The obtained results from this study suggested that HGinduced abnormal lipid storage was attenuated in the presence of BLE. This in vivo anti-lipogenic effect of BLE might be contributed to decreased glucose levels in cytosol and consequent reduction in the IIS pathway signal. In addition, previous studies suggested that excessive ROS production could lead to fat accumulation in C. elegans, and thus, BLE's antioxidant potential might also be responsible for a reduction in HG-induced lipogenesis of worms (25).

Several papers have been published regarding the possible therapeutic potential of bamboo species under hyperglycemic conditions. Jung's group revealed that black bamboo could suppress AGEs formation and aldose reductase activities via mediating polyol pathway (11). Studies on diabetic complications such as diabetic nephropathy, diabetic retinopathy, and diabetes-induced cardiac fibrosis were also reported $(12,26,27)$. In addition to these previous studies, our findings strongly suggest that the leaves of black bamboo have protective effects against glucose toxicity and can be a good candidate for developing anti-diabetic drugs.

\section{Conclusion}

Herein, we showed that BLE could prolong lifespan and protect worms against HG-induced toxicity via DAF16 activation. BLE also efficiently attenuated the body glucose levels, ROS production, and lipid accumulation under HG condition. Therefore, it would be interesting to conduct additional studies characterizing BLE's active compounds and elucidating detailed mechanisms.

\section{Author's contributions}

DSC designed the experiment and wrote the manuscript. EHP performed experiments. HJK gave technical support and advice. All authors confirmed the final version for submission and approved the publication.

\section{Conflict of interests}

The authors declare no conflict of interest.

\section{Ethical considerations}

All authors carefully checked the ethical issues including plagiarism, misconduct, data fabrication, and falsification, double publication or submission redundancy.

\section{Funding/Support}

None.

\section{References}

1. Roden M. [Diabetes mellitus: definition, classification and diagnosis]. Wien Klin Wochenschr. 2016;128 Suppl 2:S3740. doi: 10.1007/s00508-015-0931-3.

2. Rolo AP, Palmeira CM. Diabetes and mitochondrial function: role of hyperglycemia and oxidative stress. Toxicol Appl Pharmacol. 2006;212(2):167-78. doi: 10.1016/j. taap.2006.01.003.

3. Brownlee M. Biochemistry and molecular cell biology of diabetic complications. Nature. 2001;414(6865):813-20. doi: $10.1038 / 414813 \mathrm{a}$.

4. Montague CT, O'Rahilly S. The perils of portliness: causes and consequences of visceral adiposity. Diabetes. 2000;49(6):883-8. doi: 10.2337/diabetes.49.6.883.

5. Camp HS, Ren D, Leff T. Adipogenesis and fat-cell function in obesity and diabetes. Trends Mol Med. 2002;8(9):442-7. doi: 10.1016/s1471-4914(02)02396-1.

6. Hardy OT, Czech MP, Corvera S. What causes the insulin resistance underlying obesity? Curr Opin Endocrinol Diabetes Obes. 2012;19(2):81-7. doi: 10.1097/ MED.0b013e3283514e13.

7. Shang YF, Kim SM, Um BH. Optimisation of pressurised liquid extraction of antioxidants from black bamboo leaves. Food Chem. 2014;154:164-70. doi: 10.1016/j. foodchem.2013.12.050.

8. Yang JH, Choi MH, Na CS, Cho SS, Kim JH, Ku SK, et al. Bamboo stems (Phyllostachys nigra variety henosis) 
containing polyphenol mixtures activate Nrf2 and attenuate phenylhydrazine-induced oxidative stress and liver injury. Nutrients. 2019;11(1). doi: 10.3390/nu11010114.

9. Choi MH, Jo HG, Yang JH, Ki SH, Shin HJ. Antioxidative and anti-melanogenic activities of bamboo stems (Phyllostachys nigra variety henosis) via PKA/CREB-mediated MITF downregulation in B16F10 melanoma cells. Int J Mol Sci. 2018;19(2). doi: 10.3390/ijms19020409.

10. Van Hoyweghen L, De Bosscher K, Haegeman G, Deforce $D$, Heyerick A. In vitro inhibition of the transcription factor NF- $\kappa \mathrm{B}$ and cyclooxygenase by bamboo extracts. Phytother Res. 2014;28(2):224-30. doi: 10.1002/ptr.4978.

11. Jung SH, Lee JM, Lee HJ, Kim CY, Lee EH, Um BH. Aldose reductase and advanced glycation endproducts inhibitory effect of Phyllostachys nigra. Biol Pharm Bull. 2007;30(8):1569-72. doi: 10.1248/bpb.30.1569.

12. Ying C, Mao Y, Chen L, Wang S, Ling H, Li W, et al. Bamboo leaf extract ameliorates diabetic nephropathy through activating the AKT signaling pathway in rats. Int J Biol Macromol. 2017;105(Pt 3):1587-94. doi: 10.1016/j. ijbiomac.2017.03.124

13. Lithgow GJ, White TM, Melov S, Johnson TE. Thermotolerance and extended life-span conferred by single-gene mutations and induced by thermal stress. Proc Natl Acad Sci U S A. 1995;92(16):7540-4. doi: 10.1073/ pnas.92.16.7540.

14. Yoon DS, Lee MH, Cha DS. Measurement of intracellular ROS in Caenorhabditis elegans using 2',7'-dichlorodihydrofluorescein diacetate. Bio Protoc. 2018;8(6). doi: 10.21769/BioProtoc. 2774

15. Lee SJ, Murphy CT, Kenyon C. Glucose shortens the life span of C. elegans by downregulating DAF-16/FOXO activity and aquaporin gene expression. Cell Metab. 2009;10(5):379-91. doi: 10.1016/j.cmet.2009.10.003.

16. Garcia AM, Ladage ML, Dumesnil DR, Zaman K, Shulaev V, Azad RK, et al. Glucose induces sensitivity to oxygen deprivation and modulates insulin/IGF-1 signaling and lipid biosynthesis in Caenorhabditis elegans. Genetics. 2015;200(1):167-84. doi: 10.1534/genetics.115.174631.

17. Habeos I, Papavassiliou AG. Type 2 diabetes mellitus and worm longevity: a transcriptional link to cure? Trends Endocrinol Metab. 2001;12(4):139-40. doi: 10.1016/s10432760(01)00401-5.
18. Liggett MR, Hoy MJ, Mastroianni M, Mondoux MA. Highglucose diets have sex-specific effects on aging in C. elegans: toxic to hermaphrodites but beneficial to males. Aging (Albany NY). 2015;7(6):383-8. doi: 10.18632/aging.100759.

19. Choi SS. High glucose diets shorten lifespan of Caenorhabditis elegans via ectopic apoptosis induction. Nutr Res Pract. 2011;5(3):214-8. doi: 10.4162/nrp.2011.5.3.214.

20. Salih DA, Brunet A. FoxO transcription factors in the maintenance of cellular homeostasis during aging. Curr Opin Cell Biol. 2008;20(2):126-36. doi: 10.1016/j. ceb.2008.02.005.

21. Schlotterer A, Kukudov G, Bozorgmehr F, Hutter H, Du $\mathrm{X}$, Oikonomou D, et al. C. elegans as model for the study of high glucose- mediated life span reduction. Diabetes. 2009;58(11):2450-6. doi: 10.2337/db09-0567.

22. Wang $\mathrm{X}$, Desai $\mathrm{K}$, Clausen JT, Wu L. Increased methylglyoxal and advanced glycation end products in kidney from spontaneously hypertensive rats. Kidney Int. 2004;66(6):2315-21. doi: 10.1111/j.1523-1755.2004.66034.x.

23. Ye R, Onodera T, Scherer PE. Lipotoxicity and $\beta$ cell maintenance in obesity and type 2 diabetes. J Endocr Soc. 2019;3(3):617-31. doi: 10.1210/js.2018-00372.

24. Schulz TJ, Zarse K, Voigt A, Urban N, Birringer M, Ristow M. Glucose restriction extends Caenorhabditis elegans life span by inducing mitochondrial respiration and increasing oxidative stress. Cell Metab. 2007;6(4):280-93. doi: 10.1016/j.cmet.2007.08.011.

25. Wang $\mathrm{K}$, Chen $\mathrm{S}$, Zhang $\mathrm{C}$, Huang J, Wu J, Zhou H, et al. Enhanced ROS production leads to excessive fat accumulation through DAF-16 in Caenorhabditis elegans. Exp Gerontol. 2018;112:20-9. doi: 10.1016/j. exger.2018.07.017.

26. Lee HJ, Kim KA, Kang KD, Lee EH, Kim CY, Um BH, et al. The compound isolated from the leaves of Phyllostachys nigra protects oxidative stress-induced retinal ganglion cells death. Food Chem Toxicol. 2010;48(6):1721-7. doi: 10.1016/j.fct.2010.03.052.

27. Zhang L, Mao Y, Pan J, Wang S, Chen L, Xiang J. Bamboo leaf extract ameliorates cardiac fibrosis possibly via alleviating inflammation, oxidative stress and apoptosis. Biomed Pharmacother. 2017;95:808-17. doi: 10.1016/j. biopha.2017.08.138. 